\title{
NOUVELLE
}

\section{Les molécules qui dirigent la migration des astrocytes}

Sandrine Etienne-Manneville ou par les leucocytes infiltrés. Cependant, les mécanismes et le contrôle de la migration astrocy-

MRC Laboratory of Molecular Cell Biology, University College London, Gower Street, London WClE 6BT, Royaume-Uni.

taire sont toujours mal connus.

\section{Un modèle de migration} astrocytaire in vitro

Les astrocytes peuvent être isolés et
$>$ Les astrocytes représentent une grande majorité des cellules gliales et, en conséquence, occupent une place prépondérante au sein du système nerveux central.

Les astrocytes, des cellules essentielles du système nerveux central

Longtemps considérées comme un simple support physique des neurones, les astrocytes remplissent en fait de très nombreuses fonctions nécessaires au développement et au bon fonctionnement du système nerveux. Ils jouent un rôle essentiel dans la mise en place et le maintien des barrières qui limitent le système nerveux central, comme la barrière hémato-encéphalique et les méninges. Ils contrôlent aussi l'homéostasie cérébrale en réglant les concentrations en ions et en neurotransmetteurs. Enfin, ils produisent de nombreux facteurs neurotrophiques et les métabolites nécessaires au métabolisme neuronal. Les astrocytes fournissent donc les conditions idéales au développement et à la survie des neurones. Des études plus récentes ont aussi mis en évidence le rôle fondamental des astrocytes dans la formation et la stabilisation des synapses nerveuses [1]. Les astrocytes contribuent aux réponses immunes et au maintien de l'intégrité tissulaire du système nerveux central. En effet, ils peuvent produire de nombreuses cytokines et exprimer les molécules nécessaires à la présentation antigénique.

Dans les conditions physiologiques normales, chez l'adulte, les astrocytes sont des cellules immobiles, de forme étoilée sans orientation privilégiée. L'apparition d'un site d'inflammation, à la suite d'une pathologie ou d'une lésion du système nerveux, entraîne une réaction des astrocytes appelée gliose réactive [2]. La libération de facteurs inflammatoires provoque notamment une réorientation des astrocytes en direction du site d'inflammation. Les astrocytes se divisent, s'allongent et migrent en direction du site lésé jusqu'à former une cicatrice gliale. Cette cicatrice constitue une nouvelle barrière imperméable entre le système nerveux et les tissus détériorés, permettant ainsi le rétablissement de conditions propices à la régénération neuronale au sein du système nerveux. Cependant, les nouveaux axones ne peuvent pas se développer au-delà de la cicatrice, ce qui limite spatialement les capacités de régénération [3]. L'orientation et la migration des astrocytes, qui contribuent à déterminer la position de la cicatrice gliale, jouent donc un rôle essentiel dans les capacités de récupération du système nerveux après une lésion accidentelle ou pathologique. Chez les mammifères, contrairement aux vertébrés inférieurs, la migration des astrocytes est très limitée, ce qui pourrait expliquer les capacités de régénération relativement restreintes de leur système nerveux [4]. Les réponses astrocytaires semblent être réglées par des facteurs de croissance et des cytokines inflammatoires qui sont libérés par les cellules lésées (astrocytes, neurones ...), par les cellules microgliales étudiés en culture in vitro. Des études ont montré que la rayure d'une monocouche astrocytaire en culture provoque une réorientation des cellules et une migration similaires à celles observées in vivo à la suite d'une lésion $[3,5]$. Nous avons utilisé ce modèle in vitro pour caractériser la réponse astrocytaire et en particulier les mécanismes qui contrôlent la réorientation et la migration de ces cellules [6]. Dans ces conditions, la migration des astrocytes est très lente (environ 0,1 $\mathrm{mm} / \mathrm{min}$ ) en comparaison avec d'autres types cellulaires étudiés dans les mêmes conditions, ce qui traduit la capacité de migration limitée des astrocytes observée in vivo. En revanche, la migration est très polarisée (Figure 1) : les cellules s'orientent, s'allongent en formant une protrusion longue d'une centaine de micromètres, et migrent dans la direction perpendiculaire à la rayure initiale ; elles finissent par refermer l'espace créé par la rayure.

\section{Le rôle du cytosquelette dans la migration astrocytaire} Le cytosquelette cellulaire, comprenant les microfilaments d'actine, les microtubules et les filaments intermédiaires, sous-tend les changements morphologiques et la motilité cellulaire. Dans le cas des fibroblastes ou des leucocytes, le cytosquelette d'actine est l'acteur essentiel de la migration cellulaire et les microtubules ne jouent qu'un rôle mineur. Cependant, dans le cas de la migration des astrocytes, nous avons pu montrer que les microtubules jouent aussi un rôle fondamental. En effet, des inhibiteurs des microtubules bloquent totalement la migration astrocytaire, et empèchent la formation des protrusions et la réorientation des cellules dans le sens de 
la migration. De plus, la visualisation des microtubules par immunofluorescence montre leur réorganisation importante : d'une part, ils s'orientent dans le sens de la migration et, d'autre part, ils s'allongent et remplissent la protrusion. Le centre organisateur des microtubules (MTOC) lui aussi se réoriente en réponse à la lésion, en se plaçant en avant du noyau cellulaire dans le sens de la migration (Figure 1).

\section{Les acteurs de la réorganisation des microtubules}

De nombreux acteurs de la régulation du cytosquelette d'actine sont connus. En particulier, les petites protéines $G$ de la famille Rho (Rho, Rac et $\mathrm{Cdc} 42$ ) gouvernent la formation de structures d'actine [7], nécessaires à la migration des fibroblastes [8]. La régulation de la dynamique des microtubules est moins bien connue que celle de l'actine. Nous nous sommes donc intéressés au rôle des petites protéines $G$ de la famille Rho dans la polarisation des astrocytes et la formation des protrusions astrocytaires, phénomènes qui dépendent des microtubules et non de l'actine. Le modèle in vitro de migration astrocytaire a permis de montrer que l'activité de Cdc42 est nécessaire à la réorientation des microtubules observée en réponse à la lésion. Cdc42 joue aussi un rôle dans la formation des protrusions, mais ces dernières dépendent avant tout de Rac. Ces résultats, ainsi que de récents travaux montrant le rôle de Rho dans la stabilisation des microtubules [9], indiquent que les petites protéines $G$ de la famille Rho peuvent régler non seulement le cytosquelette d'actine mais aussi les microtubules. Parmi les différents effecteurs potentiels de Cdc42 [10], nous avons mis en évidence que la protéine Par6 et $\mathrm{PKC} \zeta$, une protéine kinase C atypique [11], contrôlent la réorientation des microtubules et du MTOC sans être nécessaires à la formation des protrusions (Figure 2). $\varepsilon$ n revanche, Rac, qui est nécessaire à la formation des protrusions, n'est pas requis pour la réorientation des microtubules et du MTOC. Ces résultats indiquent que les mécanismes moléculaires qui contrôlent la polarisation cellulaire et la formation des protrusions lors du déclenchement de la migration sont relativement indépendants.

\section{Les signaux déclenchant}

\section{la migration des astrocytes}

Les petites protéines $G$ de la famille Rho jouent donc un rôle essentiel dans l'induction de la migration des astrocytes. Ces protéines interviennent dans de nombreuses voies de signalisation et peuvent être activées en réponse à un grand nombre de stimulus. La lésion d'une monocouche cellulaire est susceptible de provoquer nombre de ces stimulus, tels que la libération de molécules cytoplasmiques, comme I'ATP, par les cellules lésées, l'ouverture des jonctions intercellulaires sur le front de la lésion, ou encore la formation d'un espace libre devant les cellules qui devront migrer. Parmi ces différents stimulus, nous avons pu montrer que la création d'un espace libre devant les cellules qui bordent la lésion est un élément essentiel à l'activation des voies de signalisation conduisant à la polarisation et à la migration cellulaire. En effet, l'élimination des cellules à l'emplacement de la lésion rend accessible la matrice extracelmigrent dans la direction perpendiculaire à la rayure (barre : $50 \mathrm{~nm}$ ). 
lulaire. Les cellules du bord de la lésion peuvent adhérer à la matrice extracellulaire par l'intermédiaire des intégrines membranaires et reconnaissent de cette façon l'espace libre. En se liant aux molécules de la matrice extracellulaire, les intégrines transmettent un signal vers l'intérieur de la cellule conduisant notamment à l'activation de la petite protéine G, Cdc42 [12]. L'activation des intégrines a lieu essentiellement à l'avant des cellules qui bordent la lésion. Ceci permet l'activation localisée de Cdc42 nécessaire à la polarisation et la migration des astrocytes (Figure 2).

\section{Conclusions}

La migration des astrocytes est un phénomène extrêmement contrôlé qui met en jeu plusieurs voies de signalisation aboutissant d'une part à la réorientation des cellules et, d'autre part, à des changements morphologiques suivis par la migration proprement dite des astrocytes. De nombreuses questions restent à résoudre. Quelle est la nature des intermédiaires qui permettent l'activation de Cdc42 par les intégrines? Quels sont les éléments moléculaires qui contrôlent directement la polarisation des microtubules en réponse à l'activité de $\mathrm{PKC \zeta}$. Des mécanismes moléculaires similaires sontils impliqués lorsque les astrocytes ne répondent pas à une lésion mais à des facteurs inflammatoires libérés à distance au cours de situations pathologiques? Tester in vivo les résultats obtenus grâce à ce modèle in vitro de migration astrocytaire permet d'envisager une compréhension plus fine des réponses astrocytaires aux pathologies cérébrales. Enfin, l'étude de la migration astrocytaire devrait permettre une meilleure connaissance des gliomes, tumeurs cérébrales dérivées d'astrocytes. Ces tumeurs sont particulièrement invasives, ce qui les rend réfractaires à de nombreuses approches thérapeutiques classiques. Comparer les mécanismes de régulation de la migration d'astrocytes normaux à ceux impliqués dans la migration beaucoup plus rapide, non contrôlée et non orientée des cellules de gliomes pourrait permettre de développer de nouvelles stratégies thérapeutiques ciblant les tumeurs cérébrales. $\downarrow$

Mechanisms underlying polarised

migration of astrocytes
1. Ullian EM, Sapperstein SK, Christopherson KS, Barres BA. Control of synapse 2001; 291: 657-60.

2. Ridet JL, Malhotra SK, Privat A, Cage FH. Reactive astrocytes: cellular and molecular cues to biological function. Trends Neurosci 1997; 20: 570-7.

3. Shearer MC, Fawcett JW. The astrocyte/meningeal cell interface-a barrier to successful nerve regeneration? Cell Tissue Res 2001; 305: 267-73.

4. Sivron T, Schwartz M. Glial cell types, lineages and response to injury in rat and fish: implication for regeneration. Glia 1995 . 13: 157-65. number by glia. Science
5. Faber-Elman A, Solomon A, Abraham JA, Marikovsky M, Schwartz M. Involvement of wound-associated factors in rat brain astrocyte migratory response to axonal injury : in vitro simulation. J Clin Invest 1996; 97: 162-71.

6. Etienne-Manneville $S$, Hall S. Integrin-mediated $\mathrm{Cdc} 42$ activation controls cell polarity in migrating astrocytes through PKCz. Cell 2001; 106: 489-98.

7. Hall A. Rho GTPases and the actin cytoskeleton. Science 1998; 279: 509-14.

8. Nobes CD, Hall A. Rho GTPases control polarity, protrusion and adhesion during cell movement. J Cell Biol 1999; 144: 1235-44.
9. Ishizaki T, Morishima Y, Okamoto M, Furuyashiki T, Kato T, Narumiya S. Coordination of microtubules and actin effector mDial. Nat Cell Biol 2001; 3: 8-14.

10. Bishop AL, Hall A. Rho GTPases and their effector proteins. Biochem / 2000; 348: 241-55.

11. Joberty G, Petersen C, Gao $L$, Macara IG. The cellpolarity protein Par6 links Par3 and atypical protein kinase $\mathrm{C}$ to $\mathrm{Cdc} 42$. Nat Cell Biol 2000; 2: 531-9.

12. Ridley A. Rho GTPases: integrating integrin signaling. J Cell Biol 2000; 150: F107-9. cytoskeleton by the Rho

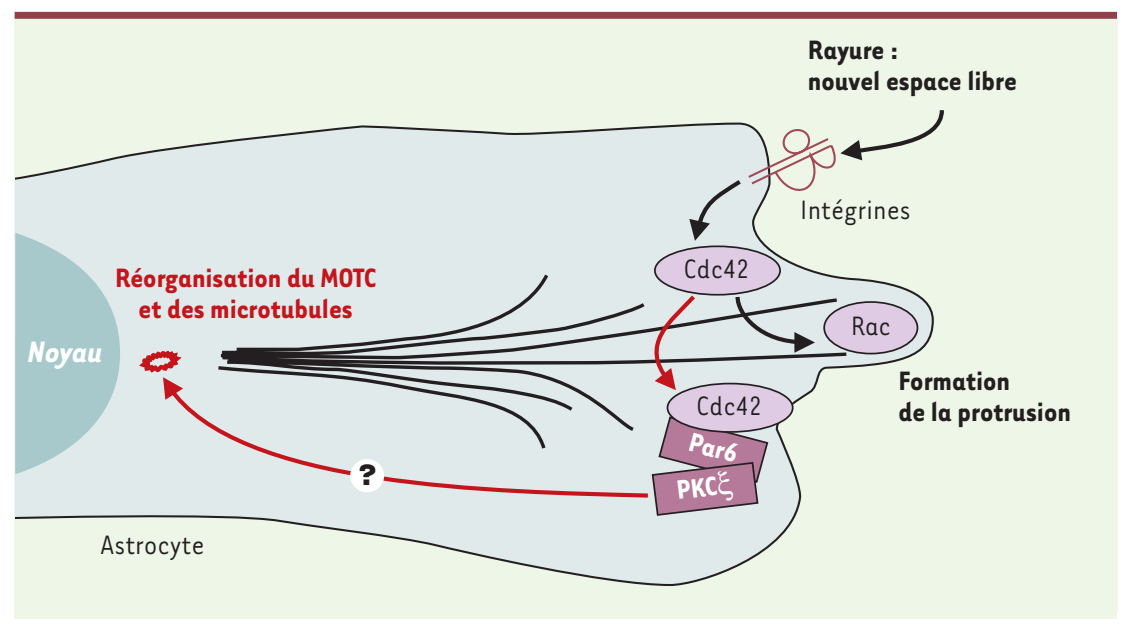

Figure 2. Mécanismes moléculaires responsables du déclenchement de la migration des astrocytes. À la suite de la rayure de la monocouche, l'espace libre créé à l'avant des cellules qui bordent la lésion stimule les intégrines membranaires, qui transmettent alors un signal intracellulaire conduisant à l'activation de Cdc42. Cdc42 est impliquée dans deux phénomènes indépendants : la formation de la protrusion, qui dépend aussi de la petite protéine $G$ Rac, et la réorientation des microtubules et du centre organisateur des microtubules (MTOC) qui dépend de Par6 et de la PKC $\zeta$. 